\title{
Evaluation of Best First Decision Tree on Categorical Soil Survey Data for Land Capability Classification
}

\author{
Nirmal Kumar \\ National Bureau of Soil Survey \\ and Land Use Planning \\ Amravati Road \\ Nagpur, Maharashtra - 440033
}

\author{
G. P. Obi Reddy \\ National Bureau of Soil Survey \\ and Land Use Planning \\ Amravati Road \\ Nagpur, Maharashtra - 440033
}

\author{
S Chatterji \\ National Bureau of Soil Survey \\ and Land Use Planning \\ Amravati Road \\ Nagpur, Maharashtra - 440033
}

\begin{abstract}
Land capability classification (LCC) of a soil map unit is sought for sustainable use, management and conservation practices. High speed, high precision and simple generating of rules by machine learning algorithms can be utilized to construct pre-defined rules for LCC of soil map units in developing decision support systems for land use planning of an area. Decision tree (DT) is one of the most popular classification algorithms currently in machine learning and data mining. Generation of Best First Tree (BF Tree) from qualitative soil survey data for LCC reported in reconnaissance soil survey data of Wardha district, Maharashtra has been demonstrated in the present study with soil depth, slope, and erosion as attributes for LCC. A 10-fold cross validation provided accuracy of $100 \%$. The results indicated that $\mathrm{BF}$ Tree algorithms had good potential in automation of LCC of soil survey data, which in turn, will help to develop decision support system to suggest suitable land use system and soil and water conservation practices.
\end{abstract}

\section{General Terms}

Data mining algorithms, Decision Tree

\section{Keywords}

Best First Decision Tree, Land Capability Classification, Information gain

\section{INTRODUCTION}

LCC - A qualitative system - developed by the US Department of Agriculture [1] is the most used land classification system. LCC provides information of the kind of soil, its location on the landscape, its extent, and its suitability for various uses, which is needed for conservation planning [2]. LCC includes eight classes of which, first four are suitable for cropland and the limitations on their use and necessity of conservation measures and careful management increase from I through IV. The remaining four classes, V through VIII, are unsuitable for cropland, but may be used for pasture, range, woodland, grazing, wildlife, recreation, and esthetic purposes. Within the broad classes are subclasses, which signify special limitations such as (e) erosion, (w) excess wetness, (s) problems in the rooting zone, and (c) climatic limitations. The task of LCC occurs every time a soil surveyor identifies a map unit. A large and diversified dataset have already been generated through soil surveys. A predefined rule set learned on these data for automatically defining the LCC of the future soil units being surveyed, will be of great help for developing decision support systems for land use planning and suggesting conservation and management practices. Machine learning and data mining techniques which gives computers the ability to learn based on the inherent characteristics of data, without being explicitly programmed $[3,4]$ may be utilized for generating these rule sets. DT is one of the most popular classification algorithms currently in machine learning and data mining [5-7].

In their simplest form, DT classifiers successively partition the input training data into more and more homogeneous sub sets by producing optimal rules or decisions, also called nodes [7-9]. The rules or the splitting criteria at these nodes are the key to successful decision tree creation. The most frequently used splitting criteria are the information gain, the information gain ratio [10], and the Gini index [11]. Some of the most popular DT methods are ID3, C4.5 [10, 12, 13], CART [11] and BF Tree [14]. A detailed review of DT applications in agricultural and biological engineering may be found in [5] and [7]. In the field of applying DT algorithms for soil survey data, [15] evaluated ID3 DT for LCC with 12 simulated samples with soil depth, slope, and texture as attributes for LCC; however, model was not validated. ID3 DT algorithm was applied on soil survey data with an accuracy of $86.84 \%$ on 10-fold cross validation [16].

\section{MATERIAL AND METHODS}

\subsection{Training Data Used}

By considering slope, soil depth and erosion as important attributes, LCC of 38 soil series of Wardha district, Maharashtra, India, was assessed as per the procedure laid down by Soil Survey Manual by All India Soil and Land Use Survey Organization [17].

Waikato Environment for Knowledge Analysis (WEKA) - an open source data mining tool - was used for generation of $\mathrm{BF}$ tree rules for comparison with the one manually generated.

\subsection{Best First Tree Algorithm}

In BF tree learners the "best" node is expanded first as compared to standard DT learners such as C4.5 and CART which expand nodes in depth-first order [14]. The "best" node is the node whose split leads to maximum reduction of impurity (e.g. Gini index or information gain) among all nodes available for splitting. The resulting tree will be the same when fully grown; just the order in which it is built is different. BF tree constructs binary trees, i.e., each internal node has exactly two outgoing edges. The tree growing method attempts to maximize within-node homogeneity. The extent to which a node does not represent a homogenous subset of cases is an indication of impurity. For example, a terminal node in which all cases have the same value for the dependent variable is a homogenous node that requires no further splitting because it is "pure." The impurity measures for nominal dependent variables are entropy-based definition of information gain and gini index. The measure used in this 
study is information gain. Entropy characterizes the purity of any sample set. If the target attribute can take on $v$ different values, then the entropy of set $(S)$ relative to this v-wise classification is defined as

$\operatorname{Entropy}(S)=\sum_{i=1}^{v}-p_{i} \log _{2} p_{i}$

where $p_{i}$ is the proportion of $S$ belonging to class i.

Information gain is the expected reduction in entropy caused by splitting the training data set according to this attribute. More precisely, the information gain, $\operatorname{Gain}(S, A)$ of an attribute A, relative to a collection of examples $S$, is defined as

$$
\begin{array}{r}
\operatorname{Gain}(S, A)=\operatorname{Entropy}(S) \\
-\sum_{v \in \text { Values }(A)} \frac{\left|S_{v}\right|}{|S|} \operatorname{Entropy}\left(S_{v}\right)
\end{array}
$$

where $\operatorname{Values}(A)$ is the set of all possible values for attribute $A$. and $S_{v}$ is the subset of $S$ for which attribute $A$ has value $v$ (i.e., $S_{v}=(S \in S|A(S)=v|)$ ).

The tree ceases to grow when all instances belong to a single value of a target feature or when best information gain is not greater than zero.

\subsection{Accuracy Assessment}

In machine learning methods, such as the DT, the classification accuracy is often predicted by stratified 10 -fold cross-validation [18-20]. In the process, the whole dataset is split into 10 parts. Nine parts of the dataset is used for learning and 1 for testing. This procedure is repeated 10 times so that every part of the dataset is used for both training and testing (of course one at each time). Afterwards, the overall accuracy parameters were calculated as means from the evaluation of the individual cross-validation subset. A 10-fold cross validation was applied in BF tree model.

\section{RESULTS AND DISCUSSION}

\subsection{Induction of BF Tree}

The LCC of soil series of the study area ranges from IIIs to Vies (Table 1). The same training dataset was used to evaluate $\mathrm{BF}$ tree in assessment of LCC.

Table 1: Soil series description and attributes for LCC

\begin{tabular}{|c|c|c|c|c|}
\hline Sirasgaon & d3 & $\mathrm{c}$ & e3 & IVes \\
\hline Talani & $\mathrm{d} 2$ & c & e3 & IVes \\
\hline Panthargavda & d3 & d & e3 & VIes \\
\hline Parsodi & $\mathrm{d} 2$ & $\mathrm{e}$ & e3 & VIes \\
\hline Hridi & $\mathrm{d} 4$ & $\mathrm{c}$ & e3 & IIIse \\
\hline Chanakpur & d3 & $\mathrm{b}$ & $\mathrm{e} 2$ & IVs \\
\hline Wadner & $\mathrm{d} 2$ & $\mathrm{~b}$ & e2 & IVs \\
\hline Pardi & $\mathrm{d} 2$ & $\mathrm{~b}$ & e2 & IVs \\
\hline Lakhandevi & $\mathrm{d} 3$ & $\mathrm{e}$ & e3 & VIes \\
\hline Mahakali & $\mathrm{d} 3$ & $\mathrm{e}$ & e3 & VIes \\
\hline Karanii & $\mathrm{d} 3$ & e & e3 & VIes \\
\hline Ashti & d3 & $\mathrm{d}$ & e3 & VIes \\
\hline Kinala & $\mathrm{d} 2$ & e & e3 & VIes \\
\hline Sewagram & $\mathrm{d} 2$ & d & e3 & VIes \\
\hline Madni & $\mathrm{d} 2$ & $\mathrm{~d}$ & e3 & VIes \\
\hline Hewan & d5 & $\mathrm{b}$ & e1 & IIIs \\
\hline Waigaon & $\mathrm{d} 4$ & $\mathrm{~b}$ & e2 & IIIse \\
\hline Karla & $\mathrm{d} 4$ & $\mathrm{~b}$ & e2 & IIIse \\
\hline Bothali & d5 & $\mathrm{b}$ & e2 & IIIse \\
\hline Kondhali & d5 & $\mathrm{b}$ & e2 & IIIse \\
\hline Wardha & d5 & $\mathrm{b}$ & e2 & IIIse \\
\hline Lasanpur & d5 & $\mathrm{b}$ & e2 & IIIse \\
\hline Malalpur & d5 & $\mathrm{b}$ & $\mathrm{e} 2$ & IIIse \\
\hline Malakpur & d5 & c & $\mathrm{e} 2$ & IIIse \\
\hline Sirpur & d5 & c & $\mathrm{e} 2$ & IIIse \\
\hline Talegaon & d5 & $\mathrm{b}$ & $\mathrm{e} 2$ & IIIse \\
\hline \multicolumn{5}{|c|}{$\begin{array}{l}\text { Depth classes: } d 2-\text { shallow }(25-50 \mathrm{~cm}), d 3-\text { moderately } \\
\text { shallow }(50-75 \mathrm{~cm}), d 4-\text { Moderately deep }(75-100 \mathrm{~cm}), d 5 \\
\text { - deep }(100-150 \mathrm{~cm}) \\
\text { Slope classes: } b-v e r y \text { gently sloping }(1-3 \%), c-\text { Gently } \\
\text { sloping }(3-8 \%), d-\text { Moderately sloping }(8-15 \%), e- \\
\text { Moderately steep }(15-30 \%) \\
\text { Erosion classes: } 1 \text { - Slight erosion, e2 - Moderate erosion, } \\
\text { e3-Severe erosion }\end{array}$} \\
\hline
\end{tabular}

\begin{tabular}{lllll}
\hline Soil series & Depth & Slope & Erosion & $\begin{array}{l}\text { Capability } \\
\text { class }\end{array}$ \\
\hline Kolona & d5 & d & e2 & IIIes \\
Karanja & d5 & d & e2 & IIIes \\
Nagjhari & d5 & d & e2 & IIIes \\
Nijampur & d5 & b & e1 & IIIs \\
Pachod & d5 & b & e2 & IIIse \\
Vagholi & d5 & b & e2 & IIIse \\
Thar & d4 & c & e2 & IIIse \\
Anjangaon & d4 & c & e2 & IIIse \\
Takli & d5 & c & e2 & IIIse \\
Arvi & d4 & b & e2 & IIIse \\
Yakamba & d4 & b & e2 & IIIse \\
Chamla & d4 & b & e1 & IIIs \\
\hline
\end{tabular}

Entropy at the root node is calculated as:

$$
\begin{gathered}
\text { entropy }(S)=-\frac{3}{38} \log _{2} \frac{3}{38}-\frac{2}{38} \log _{2} \frac{2}{38}-\frac{18}{38} \log _{2} \frac{18}{38} \\
-\frac{3}{38} \log _{2} \frac{3}{38}-\frac{3}{38} \log _{2} \frac{3}{38}-\frac{9}{38} \log _{2} \frac{9}{38}=2.094
\end{gathered}
$$

Since, the BF tree algorithm finds binary splits to split a node if the attribute erosion is considered, it has 3 possible splitting subsets (Figure 1).

In case of the first split in the figure 2, the entropy values for two successor nodes are thus

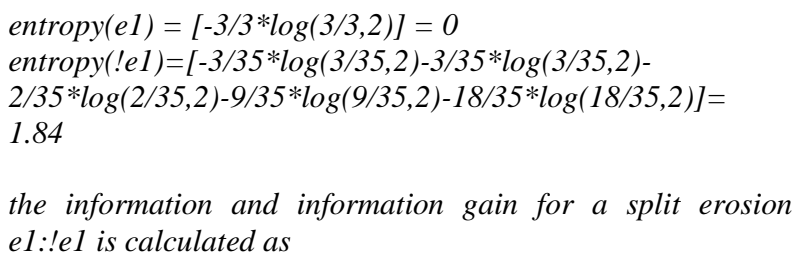

the information and information gain for a split erosion e1:!e1 is calculated as

info $($ erosion $=e 1: ! e 1)=3 / 38 *$ info $(e 1)+35 / 38 *$ info $(! e 1)=1.695$ info gain $($ erosion $=e 1: ! e 1)=$ entropy $(S)$ - info(erosion $=e 1: ! e 1)$ 

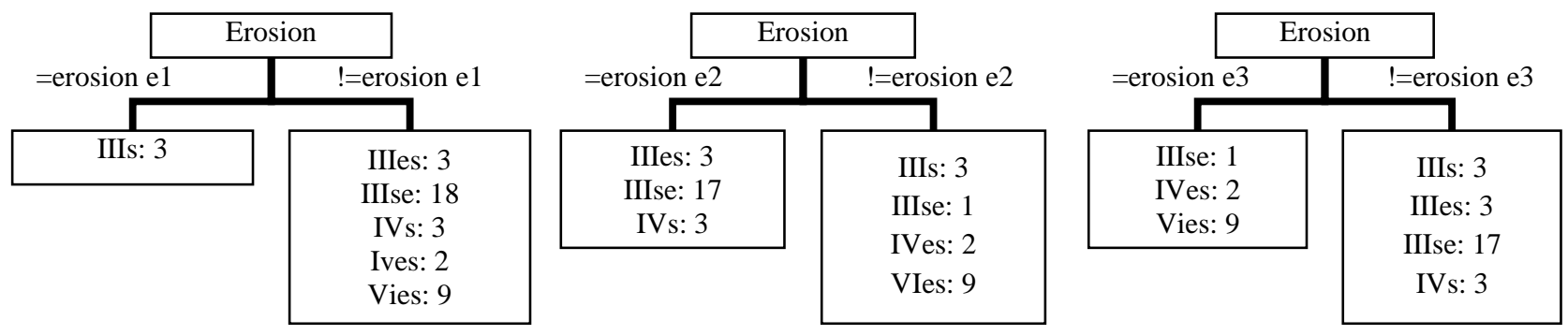

Fig 1: Possible binary splits for erosion and their class distribution at the root node

Similarly, info gains for all possible binary splits for the three attributes were calculated (Table 2). Attribute depth (binary split $d 2-3: d 4-5)$ having maximum info gain (0.949), is the choice for splitting the root node.

Table 2: Possible binary splits and info gain at the root node

\begin{tabular}{lcc}
\hline Binary Splits & info & Info gain \\
\hline Possible binary splits for depth & \\
$\mathrm{d} 2: ! \mathrm{d} 2$ & 1.765 & 0.329 \\
$\mathrm{~d} 3: ! \mathrm{d} 3$ & 1.765 & 0.329 \\
$\mathrm{~d} 4: \mathrm{d} 4$ & 1.881 & 0.213 \\
$\mathrm{~d} 5: \mathrm{d} 5$ & 1.641 & 0.453 \\
d2-3 $: \mathbf{d 4 - 5}$ & $\mathbf{1 . 1 4 4}$ & $\mathbf{0 . 9 4 9}$ \\
$\mathrm{d} 2-4: \mathrm{d} 3-5$ & 2.015 & 0.079 \\
$\mathrm{~d} 2-5: \mathrm{d} 3-4$ & 2.015 & 0.079 \\
\hline Possible binary splits for slope & \\
$\mathrm{b}: ! \mathrm{b}$ & 1.531 & 0.563 \\
$\mathrm{c}: ! \mathrm{c}$ & 1.786 & 0.308 \\
$\mathrm{~d}: ! \mathrm{d}$ & 1.639 & 0.454 \\
$\mathrm{e}: ! \mathrm{e}$ & 1.767 & 0.327 \\
$\mathrm{bc}: \mathrm{de}$ & 1.194 & 0.900 \\
$\mathrm{be}: \mathrm{cd}$ & 1.796 & 0.298 \\
$\mathrm{bd}: \mathrm{ce}$ & 1.837 & 0.257 \\
\hline Possible binary splits for & erosion & \\
$\mathrm{e} 1: \mathrm{e} 1$ & 0.600 & 0.398 \\
$\mathrm{e} 2: \mathrm{e} 2$ & 0.482 & 0.821 \\
$\mathrm{e} 3: ! \mathrm{e} 3$ & 0.492 & 0.753 \\
\hline
\end{tabular}

Child node of depth $d 2-3$ is split by slope $b$-c:d-e having maximum info gain (0.940) at this node (Table 3 ). In the same way child node of depth $d 4-5$ is split by slope $b$-c: $d$ having maximum info gain (0.544) at this node (Table 4). Node slope $d$-e under the parent node depth $d 2-3$ and slope $d$ under parent node depth $d 4-5$ is truncated as all instances belong to a single value of a target feature. Info gain at the child node slope $b-c$ (0.971) under parent node depth d2-3 (Table 5) suggests that slope $b: c$ is the next split for this node. Similarly, child node slope $b$-c under parent node depth $d 4-5$ is split by erosion e23:e1 having maximum info gain of 0.592 (Table 6). No further splitting occurs since the improvement in the gini gain comes down to zero in all child nodes.

Table 3: Possible binary splits and info gain at the child node depth $\mathrm{d} 2-3$

\begin{tabular}{lcl}
\hline Binary Splits & info & info gain \\
\hline \multicolumn{2}{l}{ Possible binary splits for slope } & \\
$\mathrm{b}: ! \mathrm{b}$ & 0.198 & 1.089 \\
$\mathrm{c}: ! \mathrm{c}$ & 0.256 & 1.031 \\
$\mathrm{~d}:$ dd & 0.391 & 0.896 \\
$\mathrm{e}:$ e & 0.362 & 0.925 \\
bc $:$ de & $\mathbf{0 . 1 2 8}$ & $\mathbf{1 . 1 5 9}$ \\
\hline
\end{tabular}

\begin{tabular}{lcc}
\hline bd : ce & 0.340 & 0.947 \\
be $:$ cd & 0.346 & 0.941 \\
\hline Possible binary splits for depth & \\
d2 : d3 & 0.466 & 0.821 \\
\hline Possible binary splits for erosion & \\
e2 $:$ e3 & 0.198 & 1.089 \\
\hline
\end{tabular}

Table 4: Possible binary splits and info gain at the child node depth $\mathrm{d4-5}$

\begin{tabular}{|c|c|c|}
\hline Binary Splits & info & info gain \\
\hline \multicolumn{3}{|c|}{ Possible binary splits for slope } \\
\hline $\mathrm{b}: \mathrm{cd}$ & 0.502 & 0.559 \\
\hline$c: b d$ & 0.593 & 0.468 \\
\hline$d: b c$ & 0.327 & 0.734 \\
\hline \multicolumn{3}{|c|}{ Possible binary splits for depth } \\
\hline $\mathrm{d} 4: \mathrm{d} 5$ & 0.619 & 0.442 \\
\hline \multicolumn{3}{|c|}{ Possible binary splits for erosion } \\
\hline e1 : !e1 & 0.327 & 0.734 \\
\hline e2 : !e2 & 0.406 & 0.655 \\
\hline e3 : !e3 & 0.659 & 0.402 \\
\hline
\end{tabular}

Table 5: Possible binary splits and info gain at the child node slope b-c under node depth $d 2-3$

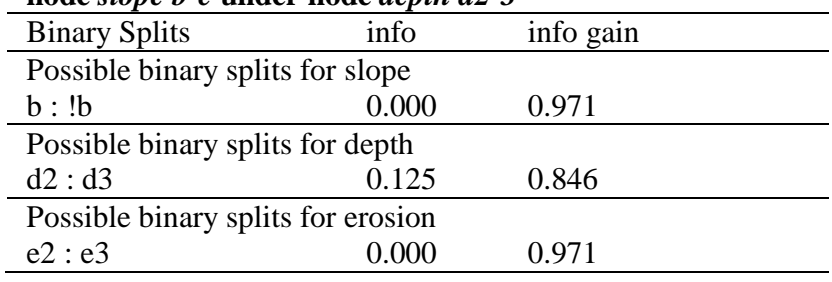

Table 6: Possible binary splits and info gain at the child node slope b-c under node depth $\mathrm{d4-5}$

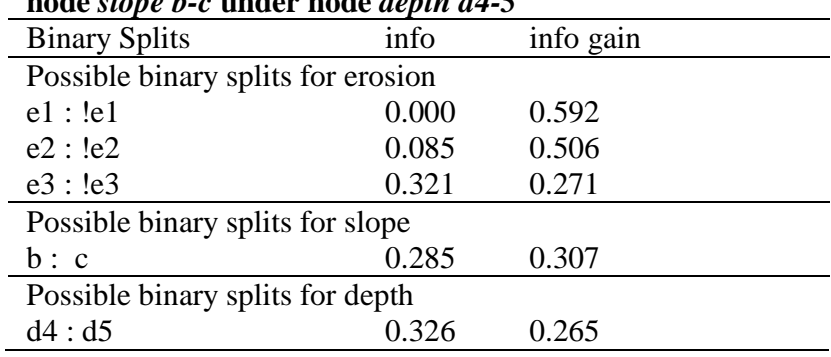

The structure of the tree manually developed is found to be similar to that of WEKA (Figure 2). The accuracy of BF tree algorithm was assessed through a 10 - fold cross validation method. The confusion matrix has been shown in table 2 . The analysis shows that BF tree algorithm found to be $100 \%$ accurate with a kappa coefficient of 1 . 


\section{CONCLUSIONS}

The result indicates that DT algorithms have immense potential over the traditional procedures in LCC for their fast and easy rules generation. Explicit rules could be formulated with better accuracy for classifying complex soil-site data acquired over diversified landscapes. The large size training dataset with higher variability may produce a robust DT model for automation of LCC and their incorporation in decision support systems to suggest suitable land use system and soil and water conservation practices.

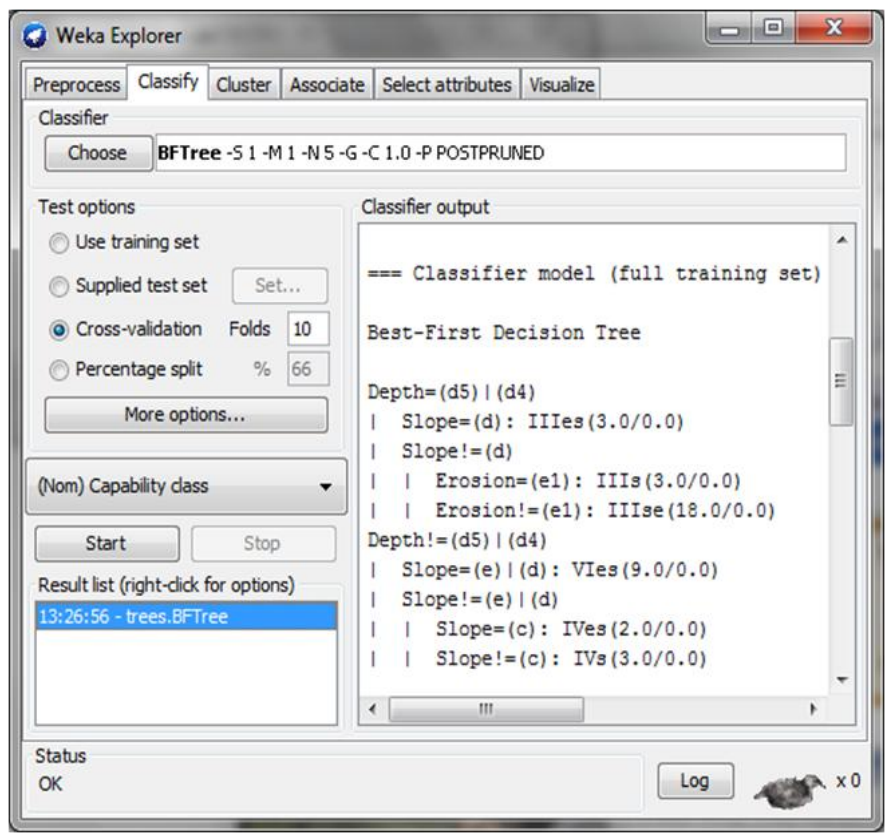

Fig. 2: Decision tree developed with BF Tree module of WEKA

Table 2: Confusion Matrix of 10 -fold cross validation in WEKA

\begin{tabular}{|l|l|l|l|l|l|l|}
\hline \multicolumn{7}{|c|}{ Confusion Matrix } \\
\hline $\mathbf{a}$ & $\mathbf{b}$ & $\mathbf{c}$ & $\mathbf{d}$ & $\mathbf{e}$ & $\mathbf{f}$ & Classified as \\
\hline $\mathbf{3}$ & 0 & 0 & 0 & 0 & 0 & a $=$ IVs \\
\hline 0 & $\mathbf{2}$ & 0 & 0 & 0 & 0 & $\mathrm{~b}=$ Ives \\
\hline 0 & 0 & $\mathbf{9}$ & 0 & 0 & 0 & c $=$ Vies \\
\hline 0 & 0 & 0 & $\mathbf{3}$ & 0 & 0 & $\mathrm{~d}=$ IIIs \\
\hline 0 & 0 & 0 & 0 & $\mathbf{1 8}$ & 0 & e = IIIse \\
\hline 0 & 0 & 0 & 0 & 0 & $\mathbf{3}$ & f = IIIes \\
\hline
\end{tabular}

\section{REFERENCES}

[1] Klingebiel, A. A., Montgomery, P. H. 1961. Land capability classification. Agriculture handbook no 210. Soil conservation service, Washington D.C. US Department of Agriculture (USDA).

[2] Fenton, T. E. 2005. Land Capability Classification. In Encyclopedia of Soil Science, Second Edition. CRC press. Pp 962-964.

[3] Fayyad, U., Piatetsky-Shapiro, G., Smyth, P., Uthuruswamy, R. (Eds.), 1996. Advances in Knowledge Discovery and Data Mining. AAAI Press, Menlo Park, CA, USA.

[4] Diplaris, S., Symeonidis, A. .L, Mitkas, P. A., Banos, G., Abasc, Z. 2006. A decision-tree-based alarming system for the validation of national genetic evaluations. Comput. Electron. Agric. 52, 21-35.

[5] McQueen, R. J., Garner, S. R., Nevill-Manning, C. G., Witten, I. H. 1995. Applying machine learning to agricultural data. Comput. Electron. Agric.12 (4), 275293.

[6] Gangrade, A., Patel, R. 2009. Building privacypreserving C4.5 decision tree classifier on multiparties. International J. Comp. Sci. Engg. 1(3), 199-205.

[7] Huang, Y., Lan, Y., Thomson S J, Fang A, Hoffmann W C, Lacey R E. 2010. Development of soft computing and applications in agricultural and biological engineering. Comput. Electron. Agric.71, 107-127.

[8] Safavian, S. R., Landgrebe, D. 1991. A survey of decision tree classifier methodology. IEEE Transactions on Systems, Man, and Cybernetics 21, 660-674.

[9] Trépos, R., Masson, V., Cordier, M. O., Chantal, G. O., Jordy, S. M. 2012. Mining simulation data by rule induction to determine critical source areas of stream water pollution by herbicides. Comput. Electron. Agric. $86,75-88$.

[10] Quinlan, J. R. 1993. C4.5: Programs for Machine Learning, Morgan Kaufmann, San Mateo, California.

[11] Breiman, L., Friedman, J. H., Olshen, R. A. 1984. Classification and Regression Trees. Belmont: Wadsworth International Group. California, USA.

[12] Quinlan, J. R. 1986. Induction of decision trees. Machine Learning 1, 81-106.

[13] Quinlan, J. R. 1996. Improved use of continuous attributes in C4.5. J. Artifi. Intel. Res. 4, 77-90.

[14] Shi, Haijian. 2007. Best-first Decision Tree Learning. Masters Degree Theses. University of Waikato Masters Theses. Pages 104.

[15] Tamboli, N. M., Kamble, A. M., Metkewar, P. S. 2012. LCC Decision tree analysis using ID3. Int. J. Comp. Appl. 41(19), 19-22.

[16] Nirmal Kumar, Obi Reddy, G. P., Chatterjee, S., Dipak Sarkar (2013). An application of ID3 decision tree algorithm for land capability classification. Agropedology. 22(1): 35-42.

[17] AISLUS. 1971. All India Soil and Land Use Survey, Soil Survey Manual, Indian Agricultural Research Institute (IARI) Publ. New Delhi.

[18] Weiss, S. M., Kulikowski, C. A. 1991. Computer systems that learn. San Mateo, CA: Morgan Kaufman Publishers.

[19] Kohavi, R. 1995. A study of cross-validation and bootstrap for accuracy estimation and model selection. Proceedings of the Fourteenth International Joint Conference on Artificial Intelligence, Montreal, Canada. Morgan Kaufmann, San Francisco, USA.

[20] Kirchner, K., T"olle, K. H., Krieter, J. 2006 Optimisation of the decision tree technique applied to simulated sow herd datasets. Comput. Electron. Agric. $50,15-24$. 